\title{
ALGUNAS PRECISIONES SOBRE EL CONCEPTO DE CECEO
}

\author{
Fco. Javier Satorre Grau. \\ Universidad de Valencia.
}

0. Las distintas acepciones que el término "ceceo" ha ido adquiriendo a lo largo de la historia de nuestra lengua han sido objeto del estudio, entre otros, de A. Alonso, R. Lapesa, D. Catalán, M. Alvar, etc. ${ }^{1}$. G. L. Guitarte se ha destacado en este campo, ya que ha estudiado este tema en diversos trabajos de reciente aparición ${ }^{2}$.

Algunas de las afirmaciones de Guitarte contradicen la tesis defendida por Alvar ${ }^{3}$. Con estas líneas pretendo simplemente aportar algunos testimonios de los siglos xvir y xviri que permitan echar un poco de luz sobre el debatido tema de qué se entendía antiguamente por "ceceo".

1 Entre los numerosos trabajos dedicados al estudio de este tema, tratan especificamente el problema de la significación del término "ceceo" los siguientes: Amado Alonso, "Historia del «ceceo» y del «seseo» españoles", en De la pronunciación medieval a la moderna en español, II, Madrid, Gredos, 1969, págs. 47-144. Rafael Lapesa, "Sobre el ceceo y el seseo andaluces", en Estudios de historia lingüística española, Madrid, Paraninfo, 1985, págs. 249-266. Diego Catalán, “El çeçeo-zezeo al comenzar la expansión atlántica de Castilla", en Boletim de Filologia, Lisboa, XVI, 1956-57 [1958], págs. 306-334. Manuel Alvar, "A vueltas con el seseo y el ceceo", en Norma lingüistica sevillana y español de América, Madrid, Ediciones de Cultura Hispánica, 1990, págs. 45-60.

2 Guillermo L. Guitarte, "Cecear y palabras afines", en Actas del II Congreso Internacional de Historia de la Lengua Española, ed. M. Ariza, R. Cano, J. M. a Mendoza y A. Narbona, Tomo I, Pabellón de España, 1992, págs. 127-164. "Los pasajes de Nebrija sobre los ceceosos", en NRFH, XXXVI, 1988, págs. 657-695. "Cecear por gracia", en Actas del VII Corgreso de la Asociación de lingüística y filología de América Latina, I, Santo Domingo, Editora UNPHU, págs. 127-141.

3 J. Mondéjar recoge la tesis de Alvar en "Disquisiciones historicocríticas y metodológicas sobre la interpretación de los datos en el estudio del «seseo»", en Actas del I Congreso Internacional sobre el Español de América, San Juan de Puerto Rico, 1982, Academia Puertorriquefía de la Lengua Española, 1987, págs. 275-276. J. A. Frago hace eco de esta diferencia de opiniones en Historia de las hablas andaluzas, Madrid, Arco, 1993, págs. 318 y siguientes. 
1. Manuel Alvar defiende que lo que los antiguos conocian por "ceceo" es un fenómeno que presentaba dos variantes indiferenciadas fonológicamente: una realización con timbre seseante (seseo), y una realización ceceante (ceceo) ${ }^{4}$. En este sentido, desarrolla la opinión de R. Lapesa de que

lo que ha variado desde el siglo xvil no ha sido la pronunciación sevillana, sino la significación de las palabras "ceceo" y "seseo". "Ceceo" ha restringido su significación, mientras "seseo" ha ampliado la suya ${ }^{5}$.

Para Lapesa, los términos con los que se designaba en el siglo xvi y primera mitad del xvit la confusión andaluza de sibilantes eran "cecear", "zezear" y "ceceo", "zezeo", nunca "sesear" ni "seseo" ".

En cambio. Guitarte niega que "cecear" pudiera tener el sentido de "sesear", simplemente por falta de pruebas. Según él, sólo se han aducido dos testinonios, ambos literarios: uno del Estebanillo González y el otro de El bachiller Trapaza, testimonios que Guitarte desmonta con unos argumentos perfectamente razonables?.

Revisando lo que dicen los diccionarios del Siglo de Oro, he podido encontrar unos textos que apoyan la teoria de Alvar y que, por lo tanto, parecen contradecir lo defendido por Guitarte.

2. El primer texto nos ofrece un testimonio totalmente claro a este respecto. Pertenece al jesuita Pedro de Salas, autor del Thesaurus Hispano Latinus, vulgarmente conocido como el Calepino de Salas. En este diccionario hispano-latino, s. v. cecear leemos: "S pro C efferre. Scibollizare. Zezissare" 8. Lamentablemente, el P. Salas no cita ningún ejemplo.

4 M. Alvar, "A vueltas con el seseo...", art. cit., pág. 50; pág. 58.

5 R. Lapesa, "Sobre el ceceo ...", art. cit., pág. 258.

- Idem, pág. 256.

7 G. L. Guitarte, “Cecear y palabras afines”, art. cit., págs. 139-140.

- La edición que poseo carece de portada, pero, tras la fe de erratas, podemos leer: "Este libro intitulado: Thesaurus Hispanolatimus, escrito por el Padre Pedro de Salas, de la Compañía de JESVS advirtiendo estas erratas, está fielmente impresso, y conforme al que otras vezes se ha dado a la estampa. Madrid, y Agosto 5 de 1688. Don Martín de Ascarça. Corrector General por su Magestad." He encontrado esta misma definición de ceceo en estas otras ediciones :

- Thesaurus Hispano Latinus.... Vallisoleti, ex Officina Bartholomaei Portoles, Uniuersitatis Typographi. Anno 1662. (Biblioteca Nacional de Madrid, 2/60180): "Cecear: $S$ pro C efferre. Scibollizare, Zesissare" (pág. 102).

- Thesaurus Hispano Latinus.... 1666. (Los datos de lugar e impresor no aparecen por estar muy estropeado el libro y faltar la parte baja de la portada.) (Biblioteca Nacional de Madrid, 3/31216): "Cecear: $S$ pro C efferre. Scibolisare. Zecissare" (página 104).

- Thesaurus hispano latinus... (Faltan las hojas del principio, donde figuran los datos 
2.1. Es bien sabido que el Thesaurus de Salas es deudor, en gran medida, del Thesaurus verborum ac phrasium del también jesuita Bartolomé Bravo ${ }^{9}$, como lo reconoce el propio Salas en las portadas de las distintas ediciones de su diccionario. Sin embargo, llama la atención que el P. Bravo no recoja en su obra la voz cecear. En cambio, si que recoge los términos çacear y çaço. El P. Bravo define çacear: "Balbutio, tis". Y çaço: "Balbus, a, um. Blaesus lingua". Estas mismas definiciones aparecen también en las voces tartamudear: "Balbutio, tis. Titubo, haereo, haesito, loqui haesitantibus verbis"; y tartamudo: "Balbus, blaesus".

El P. Salas recuge en su Thesaurus Hispano Latinus el término zazo, y lo define como "Lingua blaesus, balbus". Es decir, el P. Salas distingue perfectamente entre "zazo" y "cecear".

El P. Salas es, también, el autor de un Compendium Latino Hispanum, en el que define balbus: "el tartamudo. Hinc balbutio, is, tartamudear". Y blaesus: "el impedido de la lengua" ${ }^{10}$. De modo que parece quedar totalmente clara la diferencia que Salas establece entre "zazo" (tartamudo, torpe de lengua) y "cecear", término al que atribuye la significación de una determinada manera de pronunciar, sin que ello suponga ningún defecto en la articulación de las palabras.

El texto de Salas tiene interés porque no supone la repetición de un modelo del que está copiando, sino que, precisamente, supone una innovación con respecto al diccionario de Bravo del que en gran manera depende su obra ${ }^{11}$.

del impresor, año, licencias, censuras, etc) (Univ. de Salamanca, Biblioteca General, 50892): "Cecear: $S$ pro C efferre. Scibolizare. Zevissare" [sic] (pág. 95).

Refiriéndose al P. Salas, dice el Conde de la Viñaza: "Este P. Salas es el autor del famosísimo Diccionario Latino-español, conocido con el título de Calepino de Salas, y que fue uno de los libros más usados en las escuelas de latinidad españolas de los siglos xril y xvir." (Biblioteca histórica de la Filología Castellana, tomo tercero, Madrid, 1893. Ed. facsímil, Madrid, Atlas, 1978, pág. 938, col. 1869).

9 Thesavrvs Verborwm ac Phrasiom ad orationem ex Hispana Latinam efficiendam ct locvplendam. Auctore Bartholomaeo Bravo, Societatis IESV, Sacerdote. Multis mendis repurgatvs, exqvisitis loqvendi formulis ex Philippo Mey ... Valentiae: Typis Iacobi de Bordazar, iuxta R. Collegium Corporis Christi. Anno MDCLXXXXVIII. Expensis Ioannis Baeza. (Universidad de Valencia, Biblioteca General, Y-50/118.)

10 Compendivm Latino Hispanum vtrivsque lingvae velvti lvmen qvo Calepini Thesavri Evrici Stephani, Antonij Nebrisensis, Nizolij, P. Bartholomaei Bravo, atque omnium optimae notae Authorum labores \& locubrationes... Addimus verba sacra, ex ervditissimi viri P. Ioannis Ludouici de la Cerda, nostrae Societatis aduersariis diligenter excepta ... per Patrem Petrvm de Salas e Societate Iesv, olim in Vallisoletana vrbe publicum humanarum litterarum Professorem. Editio secunda. Ab innvmeris qvibus prior scatebat, mendis, sedvlo repurgata. Cum privilegio. Matriti. Ex typographia Bernardi a Villa-Diego, Regis ac Divini Officij Typographi. Anno. MDCXCV. (Univ. de Valencia, Biblioteca General, Y-24/40.)

11 Hay ocasiones en las que se puede constatar que el lexicógrafo recoge informaciones ajenas sin contrastarlas; incluso puede darse el caso de que se detecte que el 
2.2. El P. Salas, en la definición citada más arriba, también identifica "cecear" con "Scibollizare, Zezissare". Ninguno de estos dos vocablos aparecen recogidos en el Compendium Latino Hispanum que publica el propio jesuita madrileño, ni los he podido encontrar en ningún diccionario latino de la época o de épocas posteriores. Se trata, sin duda, de neologismos por medio de los cuales el P. Salas intenta:

a) En el caso de "Scibollizare", establecer una relación entre el fenómeno del ceceo que quiere definir, y el hecho descrito en el famoso pasaje del Libro de los Jueces, 12,6, citado tantas veces, desde Nebrija, por los gramáticos del Renacimiento, en el que los efraitas eran reconocidos por sus enemigos galaaditas por su defectuosa manera de pronunciar la palabra "Scibboleth". Para Guitarte, los pasajes de Nebrija, Aldrete, Arias Montano, etc., sobre este texto biblico no pueden interpretarse más que como una identificación de la pronunciación defectuosa de los efraítas con el "ceceo" interdental fricativo sordo ${ }^{12}$. Ignoro si el P. Salas tenía conocimientos suficientes de hebreo como para poder hacer las interpretaciones que hacen Nebrija, Aldrete y Arias sobre la pronunciación del samec en esta palabra. Lo que es indudable es que conoce la relación que los filólogos establecen entre el ceceo y el defecto de pronunciación de los de Efraím, posiblemente, a través del Tesoro de Covarrubias, diccionario que sin duda debía conocer, y en el que, curiosamente, se establece un paralelismo entre el procedimiento usado por los de Galaad para probar a los que sospechaban ser efraítas y el empleado en Castilla para reconocer a los moriscos, quienes, en vez de decir cebolla, decian sebolla, como hacían también andaluces y valencianos ${ }^{13}$.

b) Con Zexissare parece que el P. Salas está realizando una adaptación al latín de la palabra española cecear, poniendo su énfasis en la pronunciación con $\mathbf{Z}$.

3. A la luz de este texto del P. Salas puede interpretarse lo que decía L. Franciosini en su Vocabolario español $e$ italiano (1620). En este diccionario, s. v. cecear leemos: "chiamar vno facendo zi, zi con la lingua tra denti, o vero non pronunziar con buona pronunzia vna lingua, com fanno

autor desconoce la palabra que está definiendo. Así, por ej. Girolamo Vittori, en su Tesoro de las tres lenguas española, francesa y italiana (Ginebra, 1644), copia casi al pie de la letra a Oudin y recoge la palabra cacear, por zacear o çacear y, por tanto, la incluye entre las palabras que empiezan por ca- [ka] y no por las que empiezan por zao ça- (Univ. de Valencia. Biblioteca General, Y-3/24, pág. 162, que por error figura como 126.)

12 G. L. Guitarte, “Los pasajes de Nebrija ...", art. cit., págs. 659-660, 663-670 y 686-689. "Cecear y palabras afines", art. cit., págs. 138-139.

13 Sebastián de Covarrubias, Tesoro de la Lengua Castellana o Española (1611), Madrid, Turner, 1979, s. v. cecear, pág. 397. 
nella Castigliana molti forasteri \& in particolare i Sivigliani, Valenziani \& altri"14. La referencia de Franciosini a la manera defectuosa de pronunciar el castellano característica de los valencianos no admite interpretación. Los valencianos nunca han " $\theta$ e $\theta$ eado"; todo lo contrario, han pronunciado siempre una [s] ápicoalveolar que ha llamado la atención de los estudiosos de la lengua. Baste recordar simplemente la célebre cita de Jiménez Patón:

En Valencia, al contrario, y aquí no es vicio, sino natural pronunciación de aquel Reyno, por C., ponen S., como diciendo mersed, sapato, sedaso, alcusa, y assí ${ }^{15}$.

Sólo si el término cecear incluye, entre otros, los valores de pronunciar, tanto la $\mathrm{S}$ por $\mathrm{C}$, como la $\mathrm{C}$ por $\mathrm{S}$, puede comprenderse el texto del gramático y lexicógrafo italiano.

También Covarrubias (1611) asocia la pronunciación de la $\mathrm{C}$ como $\mathrm{S}$ con la manera de pronunciar de andaluces y valencianos. Al hablar de la palabra cebolla, dice Cuvarrubias:

Con este vocablo pruevan a los que sospechan ser moriscos porque pronuncian sebolla, y aun los andaluzes y valencianos y gente de cerca de la mar ${ }^{16}$.

Juan Villar (1651) titula el capítulo XI del tratado tercero de su Arte de la lengua española: "De los daños del zezear y sesear y de sus remedios". $\mathrm{Si}$ tenemos en cuenta los epígrafes que van escritos al margen del texto, parece que el $P$. Villar indica que los andaluces occidentales cecean, en tanto que los valencianos sesean. Así lo interpreta $R$. Lapesa ${ }^{17}$. Sin embargo, el P. Villar, al tratar del ceceo andaluz, se refiere tanto al trueque de $\mathrm{C}$ por $\mathrm{S}$, como al de $\mathrm{S}$ por $\mathrm{C}$ :

No es pequeño el daño que la integridad con que nuestra lengua de España en todos sus Reynos se deviera hablar padece por el zecear en esta parte Occidental de el Andalucía, donde comúnmente mudando las cees en eses, por dezir caça dizen casa, por cebo, sebo, por caço, caso, por maça, masa, y assí de sus semejantes; y por el contrario, las eses convierten en cees, como si por dezir casa, dixésemos caça, y assí de las demás ${ }^{18}$.

14 Lorenzo Franciosini, Vocabolario español $e$ italiano aora nvevamente sacado a lvz y compvesto por Lorenzo Franciosini Florentín. En Roma, a costa de Iuan Angel Rusinelli y Angel Manni. MDCXX. Por Iuan Pablo Prosilio. (Univ. de Valencia. Biblioteca General, Y-62/52, pág. 162.)

15 Bartolomé Jiménez Patón, Epitome de la ortografía latina y castellana, estudio y ed. de Antonio Quilis y J. Manuel Rozas, Madrid, CSIC, 1965, pág. 33.

16 S. de Covarrubias, Tesaro ..., obra citada, s. v. cebolla, pág. 397.

17 R. Lapesa, "Sobre el ceceo ...", art. cit., pág. 257.

18 Juan Villar, Arte de la Lengiva Española. Redvcida a reglas y preceptos de rigurosa gramática. Con notas y apuntamientos utilissimos para el perfeto conocimiento 
J. A. Frago estudia este texto y no vacila en interpretar que, aunque el P. Villar emplea únicamente el término "zecear", está teniendo en cuenta tanto el ceceo como el seseo por entonces usuales en diferentes regiones de Andalucia ${ }^{19}$.

4. Esta significación del término "ceceo" como pronunciación, tanto de la $\mathrm{S}$ como $\mathrm{C}$, como de la $\mathrm{C}$ como $\mathrm{S}$ viene confirmada en el Thesaurus utriusque linguae de Baltasar Henriquez (1679) ${ }^{20}$. En este diccionario hispano-latino, s. v. ceceo leemos: "c sono s, aut pro s, vel perinde ac s, seu contra, pronunciare, appellare, efferre". Para Henriquez, pues, el ceceo es tanto pronunciar la $\mathrm{C}$ con sonido de $\mathrm{S}$ (por ejemplo, decir sebolla), como pronunciarla en lugar de $\mathrm{S}$ (decir ceñor). Llama la atención en este texto el énfasis que pone Henriquez en indicar la doble dirección de la confusión fonética. No cabe la menor duda de cuál es el sentido que tiene para este lexicógrafo la palabra "ceceo".

5. Esta misma significación parece otorgar al término "cecear" el P. Terreros a finales del siglo xviri. En su Diccionario castellano con las voces de ciencias $y$ artes $(1786)^{21}$ define:

Cecenr, pronunciar la s como c, o la c como s. Danle el Fr. Grasseyer, begayer. El Lat. balbutire y el It. Balbetare; pero rigurosamente en todos estos idiomas significa tartamudear, lo cual es muy distinto del cecear, V.

Cécenr, se llama también por hablar con cierta especie de silvido: de modo que suena mucho la $\mathbf{s}$.

CecEAR, llamar blandamente. Fr. appeller quelqu'un dowcement Lat. Blande compellarc; también se dice por encomendar o dar a entender que se guarde silencio. V. Algunos dicen sesear en lugar de cecear.

Céceo. La acción de cecear o de mudar o trocar la s y la c. Fr. Grasseyement. Lat. Linguae haesitatio, litterarum s \& $c$ inversio.

Céceoso, sa, el que cecea como silvando o trocando la s y c. Fr. qui grasseye. Lat. Blaesus, a. It. Balbo.

de ésta y de la lengua latina. Por el P. Ivan Villar de la Compañía de Iesvs. Con licencia. En Valencia, por Francisco Verengel. Affo de 1651. (Biblioteca Nacional de Madrid, R/16271, pág. 143.)

19 J. A. Frago, Historia de las hablas andalusas, obra cit., pág. 324 . En la pág. 120 reproduce lo fundamental de este texto, que toma de A. Alonso, De la pron..., II, págs. 73-75.

so Baltasar Henriquez, Thesaurus utriusque lingwae hispanae et latinae omnium correctissimus. Matriti. Ex typographia Ioannis García Infançón, Anno 1697. (Biblioteca Nacional de Madrid, 3/4655, pág. 46.)

21 Esteban de Terreros y Pando, Diccionario Castellano con las voces de ciencias $y$ artes $y$ sus correspondientes en las tres lengwas francesa, latina $e$ italiana. Madrid. En la imprenta de la Viuda de Ibarra, Hijos y compañía. Tomo primero, 1786. Tomo tercero, 1788. Ed. facsímil, Madrid, Arco libros, 1987, I, pág. 390; III, pág. 480. 
Para Terreros el verbo "cecear" ya no significa tartamudear, sino pronunciar incorrectamente, trocando la $\mathrm{S}$ y la $\mathrm{C}$ en ambas direcciones. De modo que "cecear" es tanto pronunciar la S como C, como pronunciar la C como S. Es interesante la observación que hace Terreros de que "algunos dicen sesear en lugar de cecear". La palabra "sesear" no debía de ser todavía un vocablo de uso generalizado.

El término "sesear" aparece también definido en este diccionario: "pronunciar la $\mathrm{S}$ en lugar de $\mathrm{C}$ o de $Z$. Fr. Grasseyer. Lat. $S$ pro $C \&$ $c$ efferre, otros, blaeso ore loqui". El P. Terreros distingue entre "cecear" y "sesear", pero no opone tajantemente sus significaciones: la de "cecear" es mucho más extensa que la de "sesear", ya que prácticamente la incluye ${ }^{2}$.

5. Parece probado, pues, a la luz de estos textos, ya que el verbo "cecear" se empleó durante los siglos xvi, xvir y xviri con el valor de sesear, además de con el valor de Ge日ear, lo que confirma la tesis de Alvar de que,

al hablar del ceceo sevillano, podían distinguirse dos cosas, el timbre seseante de la capital o el ceceante del campo ${ }^{23}$.

El término "sesear", que ya emplea Jiménez Patón en 1611, no tendrá fortuna inmediatamente y no se impondrá definitivamente para significar la pronunciación de la $\mathrm{C}$ como $\mathrm{S}$ hasta mucho tiempo después, de modo que durante varios siglos el primitivo vocablo "cecear", además de con otros valores, se empleará también con el de trocar la $\mathrm{C}$ por la $\mathrm{S}$.

22 De los diccionarios de los siglos XVI, XVIr y XVIII que he consultado, tan sólo recogen la voz sesear, además del de Terreros, el Diccionario de Autoridades (1726), donde se opone perfectamente sesear: "Pronunciar las cc como ss al hablar. Lat. Litteram $S$ pro C pronuntiando usurpare", y cecear: "Pronunciar la s como c. Lat. Lingua haesitare. Balbutire...", y el Diccionario trilingüe del Castellano, Bascuence y Latín (1745), del P. Manuel Larramendi (Univ. de Valencia, Biblioteca General, X-21/25-26), en el que, aunque se definen los dos términos, éstos no tienen una significación opuesta: cecear: "ceceatu. Lat. Balbutire". Mientras que sesear: "Seseatu. Lat. S pro C efferre". Para el P. Larramendi, cecear seguía teniendo la significación de tartamudear, balbucir, en tanto que sesear tenía la significación de pronunciar incorrectamente la C como S.

${ }_{23}$ M. Alvar, “A vueltas con el seseo...”, art. cit., pág. 50. 